Submitted to PASP: 2015 November 20; AcCepted 2016 February 17; update 2016 February 26

Preprint typeset using $\mathrm{IAT}_{\mathrm{E}} \mathrm{X}$ style emulateapj v. 5/2/11

\title{
SUBMILLIMETER ATMOSPHERIC TRANSPARENCY AT MAUNAKEA, AT THE SOUTH POLE, AND AT CHAJNANTOR
}

\author{
SimOn J. E. RADFORD \\ Caltech Submillimeter Observatory, California Institute of Technology, \\ 111 Nowelo Street, Hilo, HI 96720 \\ and National Radio Astronomy Observatory, Tucson, AZ \\ JefFery B. Peterson \\ Department of Physics, Carnegie Mellon University \\ 5000 Forbes Avenue, Pittsburgh, PA 15213 \\ Submitted to PASP: 2015 November 20; Accepted 2016 February 17; update 2016 February 26
}

\begin{abstract}
For a systematic assessment of submillimeter observing conditions at different sites, we constructed tipping radiometers to measure the broad band atmospheric transparency in the window around $350 \mu \mathrm{m}$ wavelength. The tippers were deployed on Maunakea, Hawaii, at the South Pole, and in the vicinity of Cerro Chajnantor in northern Chile. Identical instruments permit direct comparison of these sites. Observing conditions at the South Pole and in the Chajnantor area are better than on Maunakea. Simultaneous measurements with two tippers demonstrate conditions at the summit of Cerro Chajnantor are significantly better than on the Chajnantor plateau.

Subject headings: Atmospheric effects - Instrumentation: tipping radiometers — Site testing - Astronomical instruments - Radio telescopes and equipment - Earth: atmosphere
\end{abstract}

\section{INTRODUCTION}

At submillimeter wavelengths, observations from the ground are practical at only a handful of exceptional sites because Earth's atmosphere is only partially transparent. Pressure broadened transitions of atmospheric molecules, water vapor in particular, delimit discrete spectral windows. Within these windows, the line wings both absorb incoming radiation, attenuating astronomical signals, and emit thermally, contributing to the background.

Because water vapor is the primary cause of atmospheric opacity, the best sites for submillimeter wavelength astronomy have exceptionally dry air. With a typical (exponential) scale height of $1-2 \mathrm{~km}$ (Holdaway et al. 1996; Turner et al. 2001), water is concentrated in the lower troposphere. Hence extremely dry air can be found above high altitude sites, particularly in subtropical desert regions. In addition, at the low temperatures encountered on the Antarctic interior plateau even saturated air has a very small water vapor content.

For a systematic assessment of observing conditions at different sites, we constructed four tipping radiometers (tippers) to measure the broad band atmospheric transparency in the $350 \mu \mathrm{m}$ window. The instruments

sradford@caltech.edu

jbp@cmu.edu were deployed on Maunakea, at the South Pole, and in the vicinity of Cerro Chajnantor in northern Chile (§3). This paper describes the instrument design and construction, the deployments, and the measurements; discusses the site characteristics; and compares the results with data from other instruments and with model predictions. Previous accounts of these measurements presented preliminary findings (Radford 2002; Peterson et al. 2003; Radford et al. 2008; Radford 2011).

\section{INSTRUMENT}

The tippers (Fig. 1) are built around an ambient temperature, deuterated L-alanine doped triglycine sulfate (DLATGS) pyroelectric detector with a sensitivity about $4 \%$ of the thermodynamic limit (Putley 1980). A light tight box holds the detector at the exit aperture of a compound parabolic concentrator (CPC; Welford \& Winston 1989). The detector signal is buffered by a JFET integrated into the detector package, amplified, and digitized at 200 samples s $^{-1}$.

A resonant metal mesh bandpass filter (QMC Instruments) mounted at the $\mathrm{CPC}$ entrance aperture defines the tipper's spectral response and a comounted plastic lowpass filter rejects unwanted mid-IR radiation. The IR rejection was confirmed by measurements with a laboratory spectrometer. The filter has a $103 \mathrm{GHz}$ (FWHM) passband centered at $850 \mathrm{GHz}$ (Fig. 2) that matches 


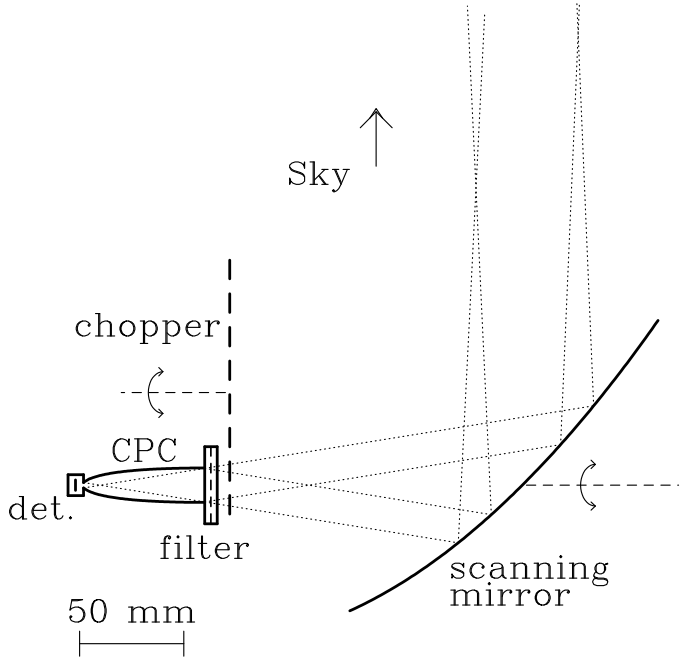

mumumumumy

calibrator

Figure 1. Optical layout of submillimeter tipper (to scale).

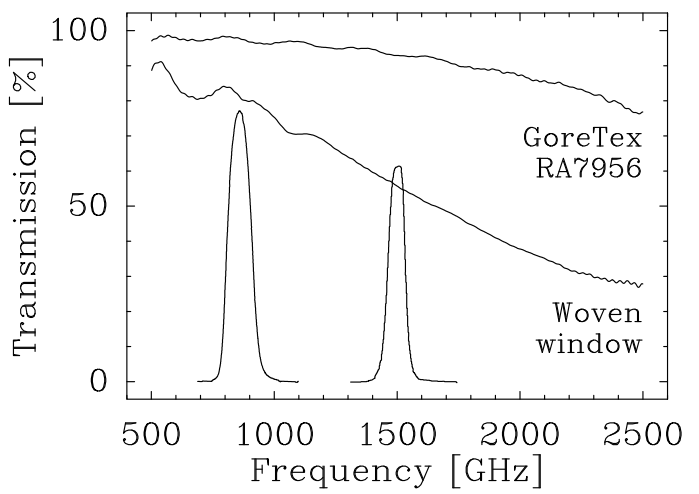

Figure 2. Submillimeter transmission of $0.25 \mathrm{~mm}$ thick woven fabric used as the tipper's weather cover window compared with unwoven GoreTex RA 7956 sheet and with passbands of $350 \mu \mathrm{m}$ and 200 mum filters. Measurements of fabric made with an spectrometer in the SAO submillimeter receiver lab (S. Paine 1998, private communication).

the $350 \mu \mathrm{m}$ atmospheric window. This passband is the same as the UKT14 bolometer (Duncan et al. 1990) and SHARC (Hunter et al. 1996; Hunter 1997; Benford 1998), is wider than SCUBA (Holland et al. 1999), but is narrower than SharcII (Dowell et al. 2003). On three tippers, the filters are fixed directly to the CPC aperture. The fourth instrument, one of the two deployed in Chile, has a filter wheel that accommodates an additional filter with an $74 \mathrm{GHz}(\mathrm{FWHM})$ passband centered at $1500 \mathrm{GHz}$ that matches the $200 \mu \mathrm{m}$ atmospheric window.

Because the detector is intrinsically differential, a rotating blade (chopper) modulates the incident radiation at the CPC entrance. The blade has three vanes to avoid even harmonics of its DC drive motor. A servo amplifier maintains the chopper at $0.75 \mathrm{~Hz}$, where the detector is most sensitive.

The CPC entrance aperture lies in the focal plane of a $90^{\circ}$ offset parabolic scanning mirror (Fig. 1). Together the CPC and the offset mirror define the tipper's $6^{\circ}$ beam (FWHM). Laboratory measurements with a transmitter
Table 1

Site Characteristics

\begin{tabular}{llcccc}
\hline \hline & & $\begin{array}{c}\text { Mauna- } \\
\text { kea }\end{array}$ & $\begin{array}{c}\text { South } \\
\text { Pole }\end{array}$ & $\begin{array}{c}\text { Chaj. } \\
\text { plateau }\end{array}$ & $\begin{array}{c}\text { Cerro } \\
\text { Chaj. }\end{array}$ \\
\hline Lat. & $\mathrm{N}$ & $19^{\circ} 48^{\prime}$ & $-90^{\circ}$ & $-23^{\circ} 1^{\prime}$ & $-22^{\circ} 59^{\prime}$ \\
Long. & $\mathrm{W}$ & $155^{\circ} 27^{\prime}$ & $\cdots$ & $67^{\circ} 45^{\prime}$ & $66^{\circ} 44^{\prime}$ \\
Noon & $\mathrm{UT}$ & $22^{\mathrm{h}} 22^{\mathrm{m}}$ & $\cdots$ & $16^{\mathrm{h}} 31^{\mathrm{m}}$ & $16^{\mathrm{h}} 31^{\mathrm{m}}$ \\
Alt. & {$[\mathrm{m}]$} & 4100 & 2835 & 5060 & $5612^{2}$ \\
$\left.T_{\text {median }}{ }^{*}{ }^{\circ} \mathrm{C}\right]$ & $2^{\mathrm{a}}$ & $-50^{\mathrm{c}}$ & $-2^{\mathrm{d}}$ & $-6^{\mathrm{e}}$ \\
WS $_{\text {med. }}{ }^{\left[\mathrm{m} \mathrm{s}^{-1}\right]}$ & $7^{\mathrm{b}}$ & $6^{\mathrm{c}}$ & $6^{\mathrm{d}}$ & $44^{\mathrm{e}}$ \\
\hline
\end{tabular}

wind speed, median

a Businger et al. 2002

b Erasmus 1986

c King \& Turner 1997

d Radford \& Holdaway 1998

e Radford et al. 2008

confirmed the beam shape. The scanning mirror rotates about the CPC axis so the detector views the sky from the zenith to the horizon or either of two calibrators. To preclude spillover onto the ground, etc., the scanning mirror and the calibrators are substantially underilluminated.

Each calibrator is made from a $12 \mathrm{~mm}$ thick slab of epoxy loaded with $5 \mu \mathrm{m}$ iron beads (Emerson \& Cuming Eccosorb MF-110). This material is the machinable form of the castable CR-110 used for several previous experiments, including the COBE mission. At $300 \mathrm{~K}$ and $350 \mu \mathrm{m}$, the specular reflectance is about $10 \%$ and the index of refraction is about 1.9 (Peterson \& Richards 1984; Hemmati et al. 1985). In the tipper calibrators, concentric triangular grooves are machined in the face of the epoxy slab. The (full) groove angle is $37^{\circ}$, so rays incident on the calibrator within $10^{\circ}$ of normal are reflected five or more times before reemerging. Moreover, the Brewster angle is $62^{\circ}$ for this material, so one polarization is almost completely absorbed. We estimate the overall specular reflectance of the calibrators is $\leq 10^{-5}$. On the sides and rear, the grooved epoxy absorber is attached to $6.4 \mathrm{~mm}$ thick aluminum plates and an electric heater is bonded to the other side of the rear plate. An analog servo circuit regulates the heater. The calibrator assembly's rear and side surfaces are insulated with $19 \mathrm{~mm}$ of polystyrene foam. The calibrator's front surface is insulated with two thin $(0.19 \mathrm{~mm})$ expanded PTFE (Zitex ZA-105) membranes suspended $3.2 \mathrm{~mm}$ and $6.4 \mathrm{~mm}$ above the calibrator surface. This material has very low attenuation throughout the submillimeter (Benford et al. 1999). One calibrator (hot) is heated to $\approx 330 \mathrm{~K}$ while the other (warm) is allowed to equilibrate with the tipper's internal temperature. On Maunakea and in Chile, the tippers' internal temperatures equilibrate about $20-25 \mathrm{~K}$ above the surroundings and follow the diurnal and seasonal cycles. At the South Pole, the tipper's internal temperature is maintained at $(284 \pm 1) \mathrm{K}$ by thermostatically controlled electric heaters.

The tipper is mounted in a weatherproof, insulated enclosure and views the sky through a window of $0.25 \mathrm{~mm}$ thick woven fabric similar to GoreTex. Across the $350 \mu \mathrm{m}$ filter passband, the window transmission is $(80 \pm 3) \%$ (Fig. 2). Across the $200 \mu \mathrm{m}$ filter passband, the window transmission is about $55 \%$. 
A computer operates the tipper, processes the data, and records the results. In particular, the synchronous demodulation and integration of the detector signal is done by software.

\section{DEPLOYMENTS}

The tippers were deployed to four premier locations for submillimeter astronomy (Table 11).

Maunakea is the highest isolated marine mountain in the world. A stable inversion layer often traps moisture below the summit, especially at night. Excellent observing conditions have led to the development of major astronomical facilities. Maunakea has become a standard of comparison for observatory sites (Morrison et al. 1973; Erasmus 1986; Businger et al. 2002). In 1997 December, a submillimeter tipper was installed pointing northwest on the roof of an outbuilding at the Caltech Submillimeter Observatory (CSO) adjacent to an existing $225 \mathrm{GHz}$ tipper. In 2016, the instrument will be relocated to the roof of the Submillimeter Array (SMA) hangar, where it will continue to point northwest.

At the South Pole the atmospheric water vapor content is very small because of the extremely low temperature even though the surface air is almost saturated (Gettelman et al. 2006). Several telescopes have been installed there, primarily for millimeter and submillimeter wavelength astronomy and cosmology (Burton et al. 2012). In 1998 January, a submillimeter tipper was installed on the roof of the AST/RO building where it pointed approximately along $135^{\circ}$ west longitude. In 2006 January, the instrument was relocated to the roof of the Dark Sector Lab (DSL) where it points along $10^{\circ}$ east longitude. An additional, fifth, instrument, with a different detector and other modifications, was deployed to Dome C, Antarctica, in 2000-1 and in 2003 with a comparison period at the South Pole in 2001-2 (Calisse et al. 2004a, b).

In northern Chile, the combined effects of a high pressure belt over the southeast Pacific, a cold offshore current, and the moisture barrier of the Andean cordillera to the east make the Atacama desert one of the driest places on Earth. The absence of glaciers, even on the highest peak in the region, Volcán Llullaillaco $(6740 \mathrm{~m})$, is unique for these altitudes (Messerli et al. 1993) and attests to the area's aridity. In recent years, the Chajantor plateau east of the village of San Pedro de Atacama has seen the installation of several radiotelescopes, culminating in the international Atacama Large Millimeter/submillimeter Array (ALMA; Beaslev 2006). In 1997 October, a submillimeter tipper was installed pointing east adjacent to an existing $225 \mathrm{GHz}$ tipper near the (then future) center of ALMA. In 2000 June, a second instrument was deployed to the same location. In 2005 May, the instruments were relocated about $1 \mathrm{~km}$ west to the site of the CBI (Padin et al. 2001) and successor experiments, where they pointed south. In 2011 November, one instrument was relocated to the APEX telescope (Güsten et al. 2006), about $4 \mathrm{~km}$ north of ALMA, again pointing south.

Several peaks rise above the Chajnantor plateau. Radiosondes launched from the plateau determined the typical (exponential) water vapor scale height is about $1.1 \mathrm{~km}$ and also revealed frequent inversion layers, especially at night, that trap moisture below the tops of these peaks (Giovanelli et al. 2001). Subsequent measurements in the submillimeter (Blundell et al. 2002; Marrone et al. 2004, 2005; Bustos et al.|2014) and in the near infrared (Konishi et al. 2015) have buttressed this result, finding exceptional observing conditions on these peaks. In 2006 May, one tipper was installed pointing south near the summit of one peak, Cerro Chajnantor, $550 \mathrm{~m}$ above and $8 \mathrm{~km}$ northeast of ALMA.

\section{MEASUREMENTS}

The tipper determines the submillimeter atmospheric transparency by the standard technique of measuring the sky brightness at several zenith angles (Dicke et al. 1946). During each tip, which takes about 13 minutes, the demodulated detector signal is integrated for $64 \mathrm{~s}$ at each of eleven positions of the scanning mirror: pointing at the hot calibrator; at the warm calibrator; at airmasses of $1,1.5,2,2.5,3,3.5$, and 4 (zenith angles of $\left.0^{\circ}, 48^{\circ}, 60^{\circ}, 66^{\circ}, 71^{\circ}, 73^{\circ}, \& 75.5^{\circ}\right)$; and at the hot and warm calibrators again. The tipper makes single sided tips from the zenith down towards one horizon. During installation the tippers were leveled within $\pm 2.5^{\circ}$.

\subsection{Calibration}

At the start and again at the end of each tip cycle, the two calibrators are observed to determine the detector responsivity,

$$
R=\left(V_{\text {hot }}-V_{\text {warm }}\right) /\left(T_{\text {hot }}-T_{\text {warm }}\right),
$$

and the effective chopper temperature,

$$
T_{\text {chop }}=T_{\text {hot }}-V_{\text {hot }} / R=T_{\text {warm }}-V_{\text {warm }} / R \text {. }
$$

Here $V_{\text {hot }}$ and $V_{\text {warm }}$ are the demodulated detector signals for the hot and warm calibrators and $T_{\text {hot }}$ and $T_{\text {warm }}$ are their physical temperatures recorded during each calibration measurement. The effective chopper temperature, $T_{\text {chop }}$, is the temperature seen by the detector when the chopper blade blocks the $\mathrm{CPC}$ entrance aperture. For each tip cycle, the average of the two calibration measurements is used to calibrate the sky measurements.

To check the calibrator performance, an absorber immersed in liquid nitrogen was substituted for the hot calibrator. The detector responsivity determined with the normal calibration and with the $\mathrm{LN}_{2}$ target agreed within $10 \%$.

Although the detector's responsivity is somewhat temperature dependent, the tipper's large thermal inertia precludes rapid changes so the transparency measurements are unaffected. Moreover, whenever there was an excessive difference between the detector responsivity measured at the start and at the end of each tip cycle, the observation was discarded.

\subsection{Window}

The tipper views the sky through a fabric window but the calibrators are inside this window. Ignoring the window would cause an underestimate of the atmospheric transparency (Calisse 2004). We model the window as an absorbing screen at the ambient exterior air temperature, $T_{\text {ext }}$. Then the brightnesses measured during a tip,

$$
\begin{aligned}
T_{\text {meas }}(A) & =\Delta V_{\text {sky }}(A) / R+T_{\text {chop }} \\
& =\theta_{\text {win }} T_{\text {sky }}(A)+T_{\text {win }}
\end{aligned}
$$




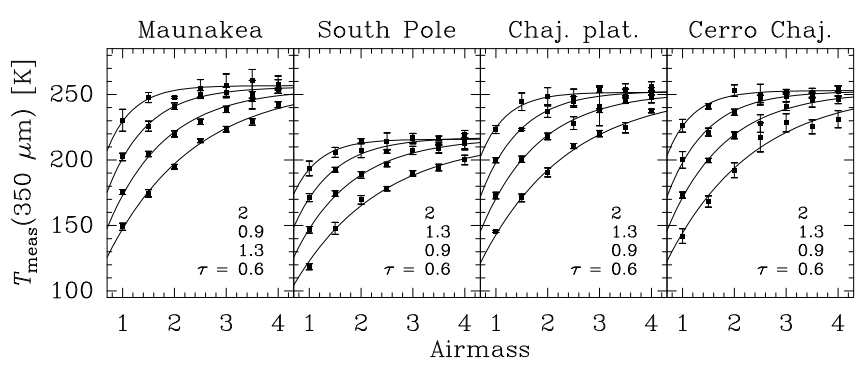

Figure 3. Representative $350 \mu \mathrm{m}$ sky brightness tips selected to show the range of conditions encountered in the measurements. They are arranged (bottom to top) in order of increasing optical depth with lines illustrating the best fit two parameter models (Eq. 4).

where $\Delta V_{\text {sky }}$ is the demodulated detector signal measured at airmass $A, T_{\text {sky }}$ is the sky brightness, $\theta_{\text {win }}$ is the window transmission, and $T_{\text {win }}=T_{\text {ext }}\left(1-\theta_{\text {win }}\right)$ is the window brightness.

To check the window correction, we temporarily placed a second layer of window material over the tipper at the South Pole while conditions were both good, $\tau \approx 0.7$, and stable. In five trials of this experiment, the apparent zenith optical depth increased by $0.18 \pm 0.07$, consistent with the calculated correction (Peterson et al. 2003).

A digital sensor (DS 1820) measured the exterior air temperature, $T_{\text {ext }}$, at the start and end of each tip cycle; the average was used for the window correction. This sensor has an absolute minimum reading of $-55^{\circ} \mathrm{C}$ $(218 \mathrm{~K})$. During the Antarctic winter the actual air temperature can fall below this. Hence the window temperature might be overestimated, leading to an overestimate of the atmospheric transparency. In practice, however, this is a small effect, less than other measurement uncertainties. If the exterior temperature were actually $-80^{\circ} \mathrm{C}$ when the sensor measures $-55^{\circ} \mathrm{C}$, the window brightness temperature would be $3 \mathrm{~K}$ colder, and the apparent optical depth would be reduced by less than $3 \%$.

\subsection{Atmospheric Transparency}

The zenith optical depth, $\tau$, is determined by fitting the measured sky brightnesses to a two parameter model of a plane parallel uniform atmosphere,

$$
\begin{aligned}
T_{\text {meas }}(A)-T_{\text {win }} & =\theta_{\text {win }} T_{\text {sky }}(A) \\
& =\theta_{\text {win }} T_{\text {atm }}\left(1-e^{-\tau A}\right),
\end{aligned}
$$

where $T_{\text {atm }}$ is the effective atmospheric brightness (Fig. 3). Typically, the uncertainty in the individual sky brightness measurements is $5 \mathrm{~K}$ or less, independent of airmass.

Because the brightness curve is non linear, the dynamic range is limited and the results are heteroscedastic: the uncertainty in the optical depth depends on the magnitude of the optical depth. The fitting procedure is robust over the range $0.5<\tau<4$ where there is sufficient curvature to separate the optical depth from the atmospheric brightness temperature. When $\tau \approx 1$, the typical uncertainty $\sigma(\tau)<0.1$. Under poor conditions, when $\tau>4$, the brightness difference between the zenith and the horizon vanishes. Then only the product $\theta_{\text {win }} T_{\text {atm }}$ can be determined and the uncertainty in optical depth diverges. A lower bound on the uncertainty is roughly $\sigma(\tau)>\tau^{4}$. For this reason, poor conditions with high optical depth
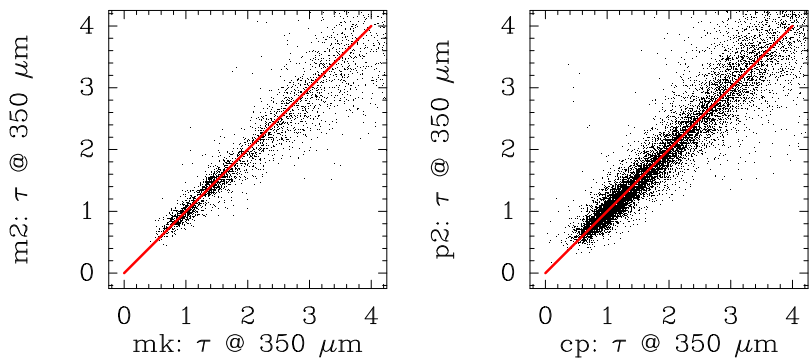

Figure 4. Correlation between broad band $350 \mu \mathrm{m}$ zenith optical depth measured simultaneously with side by side tipper pairs: (left) on Maunakea during 2011 August-October $(r=0.97)$ and (right) on the Chajnantor plateau during parts or all of 31 months in $2000-1,2005-6$, and $2009(r=0.98)$.

cannot be quantified well and must be simply regarded as poor. So long as overall statistics take this into account, this is not a practical drawback for characterizing observing conditions. (If the optical depth were very low, $\tau \ll 0.5$, the model would become linear so only the product $\theta_{\text {win }} T_{\text {atm }} \tau$ could be measured. At $350 \mu \mathrm{m}$, however, such conditions are never encountered in practice.)

Moreover, the atmosphere is not uniform so the measured sky brightness is weighted by the vertical profiles of temperature and optical depth. At large zenith angle (low elevation), the effective beam termination is lower in the atmosphere, where the air is generally warmer, than the termination at the zenith. Indeed, this effect is sometimes present in the data: near the horizon the sky temperature doesn't approach an asymptote, but continues to rise. For simplicity and because of the limited degrees of freedom in the data, we have ignored this effect in the data analysis.

\subsection{Data Edits}

At various times, the tippers suffered outages that might affect the data quality. Measurements were excised, therefore, if they failed any of several tests indicating software or hardware malfunction. The fraction of data discarded depended on local circumstances. Because these tests are based on objective, internal properties of the instruments, not on external conditions, we believe these edits have not biased the data. Indeed, the statistics of the raw, unedited data are very close to the statistics of the edited data.

\subsection{Side by side comparisons}

Although the instruments share the same design, components, and construction, side by side comparisons are important to confirm they perform identically. Although it was not possible to test all the tippers together under realistic submillimeter observing conditions prior to deployment, comparisons were made on three occasions. On Maunakea, two tippers were operated side by side during 2011 August-October. On the Chajnantor plateau, the other two instruments were operated side by side during parts or all of 31 months in 2000-1, 2005-6, and 2009. This included one week when the two instruments were separated by $1 \mathrm{~km}$, one at at ALMA and the other at the CBI. In 2001-2, the South Pole tipper was operated side by side with the modified tipper used at Dome C (Calisse et al. 2004a, b) . On all these occasions, the side by side measurements show excellent agreement 
Table 2

$350 \mu \mathrm{m}$ zenith optical depths

\begin{tabular}{lcccc}
\hline \hline & $\begin{array}{c}\text { Mauna- } \\
\text { kea }\end{array}$ & $\begin{array}{c}\text { South } \\
\text { Pole }\end{array}$ & $\begin{array}{c}\text { Chajnantor } \\
\text { plateau }\end{array}$ & $\begin{array}{c}\text { Cerro } \\
\text { Chajnantor }\end{array}$ \\
\cline { 2 - 5 } start & $1997 \mathrm{Dec}$ & 1998 Jan & $\begin{array}{c}1997 \text { Oct } \\
2016 \text { Feb }\end{array}$ & $\begin{array}{c}\text { 2006 May } \\
2013 \text { Jun }\end{array}$ \\
stop & $2016 \mathrm{Feb}$ & $2016 \mathrm{Feb}$ & 2.9 & 2.0 \\
\hline $75 \%$ & 4.2 & 1.7 & 1.7 & 1.1 \\
$50 \%$ & 2.5 & 1.3 & 1.1 & 0.8 \\
$25 \%$ & 1.5 & 1.1 & & \\
\hline
\end{tabular}

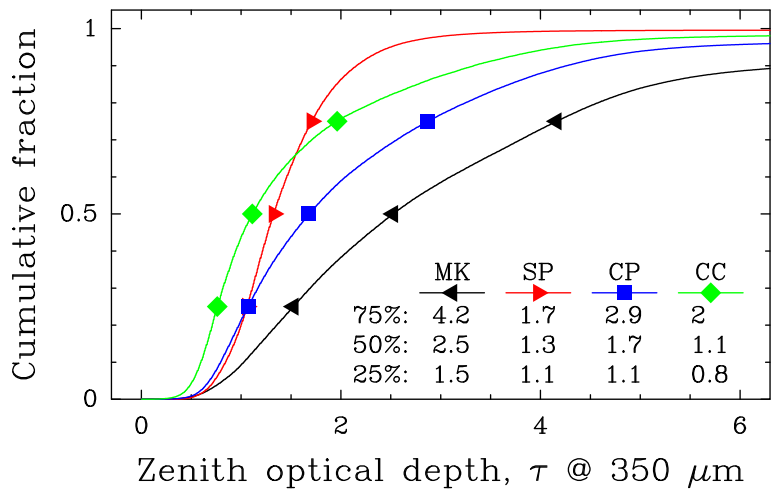

Figure 5. Cumulative distributions of broad band $350 \mu \mathrm{m}$ zenith optical depths measured on Maunakea (MK), at the South Pole (SP), on the Chajnantor plateau (CP), and on Cerro Chajnantor (CC).

(Fig. 4). The paired, simultaneous measurements are highly correlated, $r \approx 1$, and indicate the instruments produce identical results.

\section{RESULTS}

The measurements show all four sites enjoy periods of excellent observing conditions, $\tau \leq 1$ (Table 2 \& Fig. 5). Even on these occasions, however, atmospheric absorption at $350 \mu \mathrm{m}$ is substantial. As a reminder, when the zenith optical depth $\tau=1$, the zenith transmission is only $37 \%$. Submillimeter astronomy remains challenging even at premier sites.

\subsection{Site Conditions}

The best conditions occur more often at the South Pole and in the vicinity of Chajnantor than on Maunakea. The median optical depth on the Chajnantor plateau is similar to the first quartile on Maunakea. First quartile conditions at the South Pole and on the Chajnantor plateau are similar. The cumulative distribution for the South Pole is remarkably sharp; the South Pole hardly ever experiences the poor conditions, $\tau>3$, experienced at the other sites during storms. Conditions on Cerro Chajnantor are significantly better than on the the plateau.

At all locations, the minimum measured optical depth is not zero but in the range $0.3-0.4$. Although this measured minimum may be at least partially an instrumental artifact, it does depend on altitude, being smallest at the highest site, Cerro Chajnantor. This suggests the minimum corresponds to absorption by dry air, i. e., atmospheric components other than water vapor. This phenomenon has been discussed in detail previously
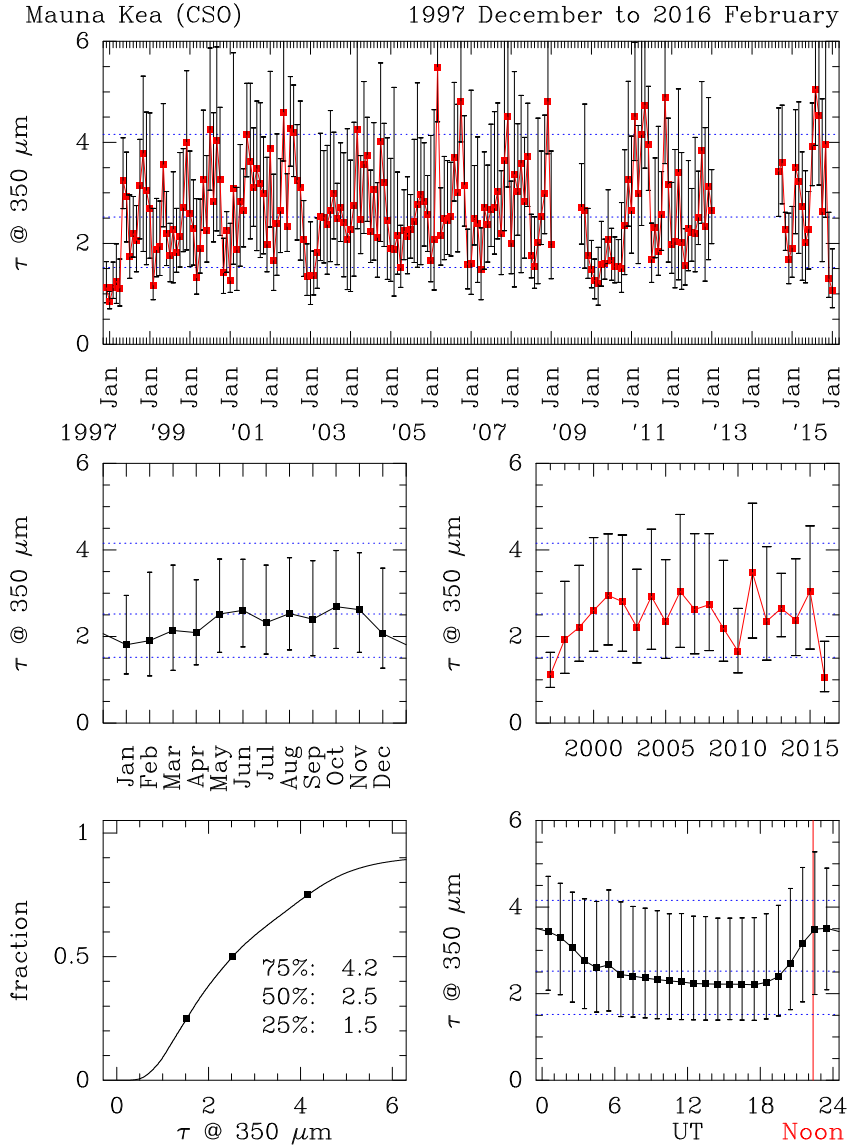

Figure 6. Broad band $350 \mu \mathrm{m}$ zenith optical depth measured on Maunakea. Top: Monthly quartiles $(25 \%, 50 \%$, \& $75 \%)$; center left: seasonal variation; center right: yearly quartiles; bottom left: cumulative distribution; bottom right: diurnal variation (with mean solar noon indicated). In each panel, markers indicate median values and the error bars show the first and third quartiles $(25 \% \& 75 \%)$. Horizontal dotted lines show the overall quartiles.

(Pardo et al. 2001a, b, 2005).

As perhaps might be expected, there is no significant correlation between the measurements at widely separated sites, i. e., between Maunakea and the South Pole or Chile. Hence the joint distribution of conditions for simultaneous observations is the product of the distributions for the individual sites. This may be a consideration, for example, for future short wavelength VLBI.

\subsection{Variations}

At all locations, the atmospheric transparency is generally better during the winter and at night. On Maunakea (Fig. 6) the seasonal pattern is evident, if not prominent. The seasonal contrast, calculated as the ratio of the maximum and minimum monthly median optical depths, is 1.5. Interannual variations are as strong as the seasonal pattern, both for the same month in different years and for entire years. At the South Pole (Fig. 7), conditions are remarkably consistent from year to year. The seasonal contrast is 1.8 , i. e., the optical depth during summer is almost twice as large as during winter. In the Chajnantor region (Figs. 8 \& 9), conditions are consistently good from April through December but deteriorate during the summer months when a shift in atmospheric circulation draws moist air over the Andes from 

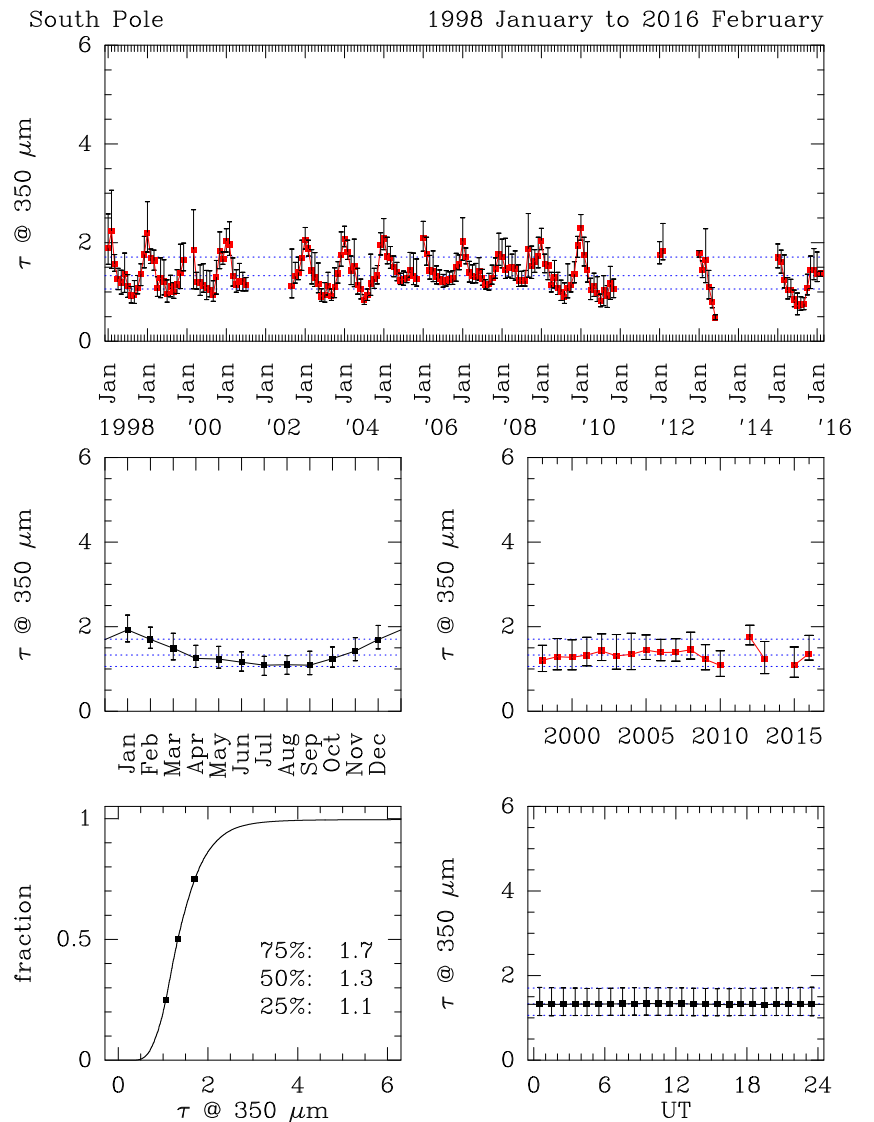

Figure 7. Broad band $350 \mu \mathrm{m}$ zenith optical depth measured at the South Pole. Top: Monthly quartiles $(25 \%, 50 \%$, \& $75 \%)$; center left: seasonal variation; center right: yearly quartiles; bottom left: cumulative distribution; bottom right: diurnal variation. In each panel, markers indicate median values and the error bars show the first and third quartiles (25\% \& $75 \%$ ). Horizontal dotted lines show the overall quartiles.

the Amazon basin. The seasonal contrast is 2.6 on the Chajnantor plateau. There is considerable interannual variation in the severity of the summer season; winter conditions are more consistent. On Cerro Chajnantor, the seasonal contrast is 3.7 , primarily because of better winter conditions.

The transparency is better at night. Both on Maunakea and in the Chajnantor area, the variation lags behind the sun; the best conditions occur around sunrise and the optical depth is highest in the afternoon. This may reflect the influence of inversion layers, or at least humid layers, that rise during the day and then subside as the night progresses. The diurnal contrast, calculated the same way as the seasonal contrast, is 1.6 at Maunakea, 1.7 on the Chajnantor plateau, and 1.5 on Cerro Chajnantor. Diurnal variations in the Chajnantor area are less pronounced during the winter than during the summer.

None of the sites display any significant secular trend in the median optical depth over the 18 year duration of the measurements. At all three locations, correlations between the monthly median optical depths and the monthly Multivariate ESNO Index (MEI; Wolter \& Timlin 2011) are insignificant $(r<0.1)$.

\subsection{Cross Comparisons}
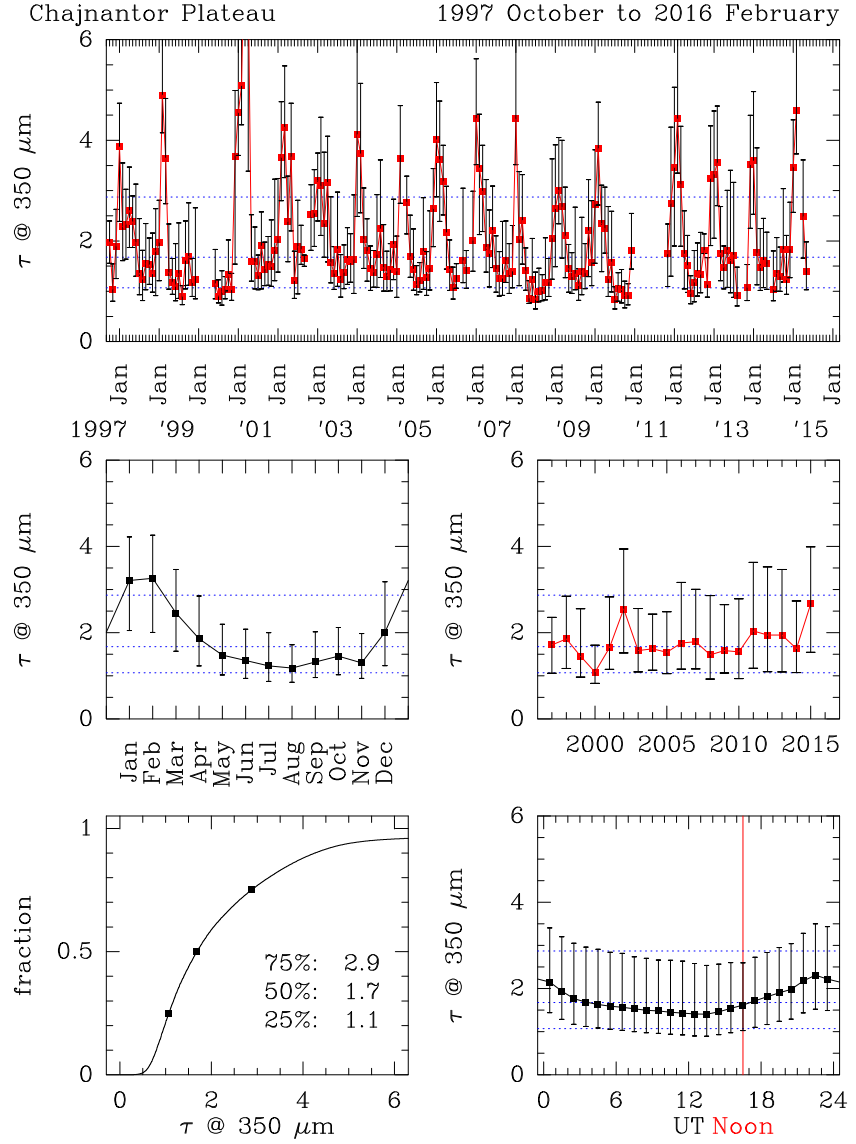

Figure 8. Broad band $350 \mu \mathrm{m}$ zenith optical depth measured on the Chajnantor plateau. These data are a non redundant composite of measurements at ALMA, at the CBI, and at APEX. Top: Monthly quartiles $(25 \%, 50 \%, \& 75 \%)$; center left: seasonal variation; center right: yearly quartiles; bottom left: cumulative distribution; bottom right: diurnal variation (with mean solar noon indicated). In each panel, markers indicate median values and the error bars show the first and third quartiles (25\% \& $75 \%)$. Horizontal dotted lines show the overall quartiles.

In Chile and on Maunakea, there were several opportunities for cross comparisons: between two tippers at nearby locations, Cerro Chajnantor and the Chajnantor plateau; between measurements made alternately at two wavelengths by a single tipper; and with other colocated instruments. In all cases, the cross comparison data are highly correlated.

Outliers are more numerous in the tipper measurements than expected for a normal distribution. To suppress the undue influence of these outliers, the data ranges considered in the regressions were restricted. In addition, data were rejected on the outskirts of the measurement distribution where the density of points fell below $2.5 \%$ of the peak. This permits a robust characterization of the range of most interest, good observing conditions.

\subsubsection{Cerro Chajnantor}

Because they are only $8 \mathrm{~km}$ apart, the two tippers on Cerro Chajnantor and on the Chajnantor plateau permit direct comparison of conditions with simultaneous measurements (Fig. 10, left). The paired measurements are highly correlated $(r>0.9)$ and indicate the $350 \mu \mathrm{m}$ at- 

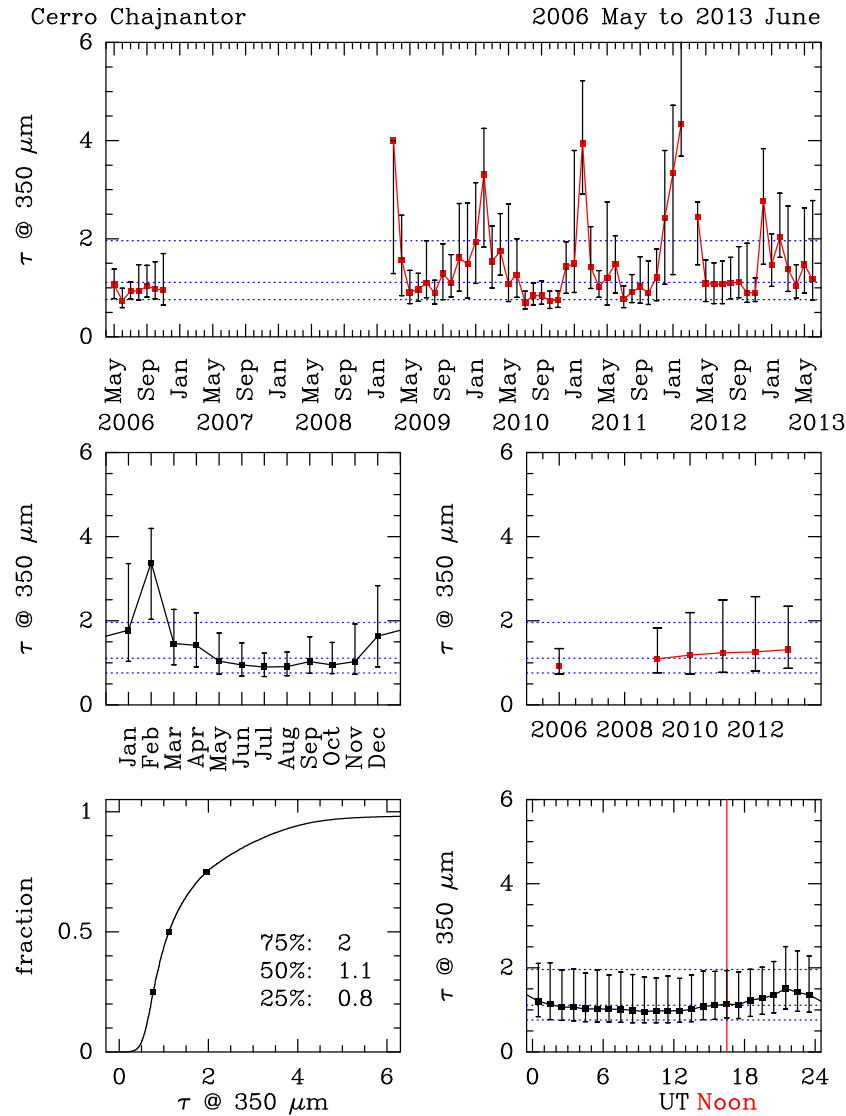

Figure 9. Broad band $350 \mu \mathrm{m}$ zenith optical depth measured on Cerro Chajnantor. Top: Monthly quartiles $(25 \%, 50 \%$, \& $75 \%)$; center left: seasonal variation; center right: yearly quartiles; bottom left: cumulative distribution; bottom right: diurnal variation (with mean solar noon indicated). In each panel, markers indicate median values and the error bars show the first and third quartiles $(25 \% \& 75 \%)$. Horizontal dotted lines show the overall quartiles.
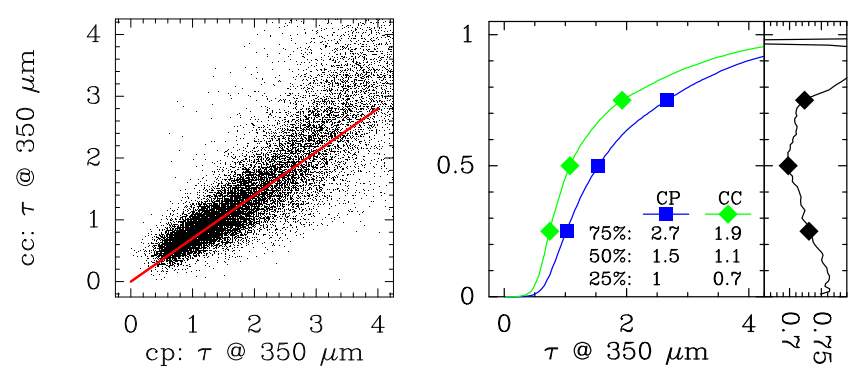

Figure 10. Left: Correlation between the broad band $350 \mu \mathrm{m}$ zenith optical depths measured simultaneously on the Chajnantor plateau (CP) and on Cerro Chajnantor (CC). The guide line shows the best linear fit $\tau_{\mathrm{cc}}=0.7 \tau_{\mathrm{cp}}$. Right: The cumulative distributions of the paired measurements and the ratio of the quantiles, $Q_{i}\left(\tau_{\mathrm{cc}}\right) / Q_{i}\left(\tau_{\mathrm{cp}}\right)$.

mospheric transparency is significantly better on Cerro Chajnantor than on the plateau. The measurement distribution is curved, which indicates the effects of saturation in the tipper measurements under poor conditions. For the best $65 \%$ of conditions $\left(\tau_{\mathrm{cp}} \leq 2.05\right.$ and $\left.\tau_{\mathrm{cc}} \leq 1.45\right)$, linear regression indicates

$$
\tau_{\mathrm{cc}}=(0.7 \pm 0.05) \tau_{\mathrm{cp}}+(0.0 \pm 0.05) \text {. }
$$
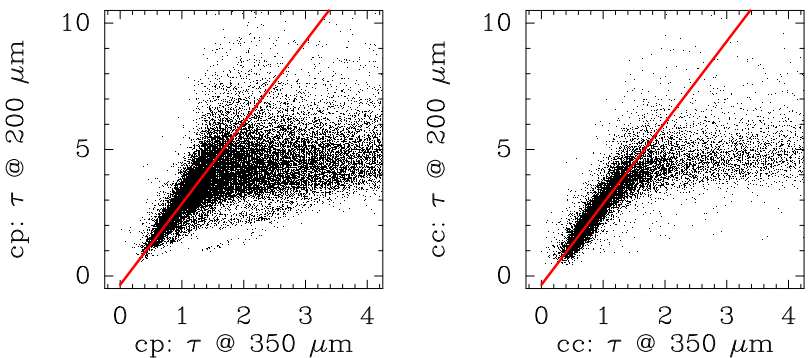

Figure 11. Correlations between successive measurements of the broad band $350 \mu \mathrm{m}$ and $200 \mu \mathrm{m}$ zenith optical depths on the Chajnantor plateau (left) and on Cerro Chajnantor (right). The measurements saturate when $\tau(200 \mu \mathrm{m})>4$. The guide lines illustrate $\tau(200 \mu \mathrm{m})=3.2 \tau(350 \mu \mathrm{m})-0.35$.
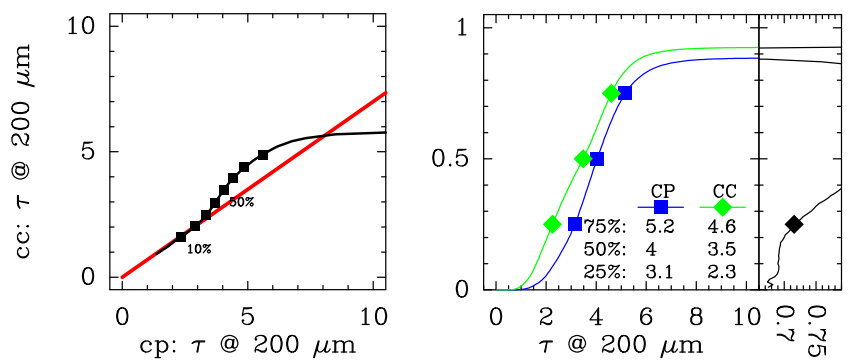

Figure 12. Left: Quantiles of the broad band $200 \mu \mathrm{m}$ zenith optical depths measured on the Chajnantor plateau $(\mathrm{CP})$ and on Cerro Chajnantor (CC) with the deciles marked $(Q Q$ plot). The measurements were not simultaneous. The guide line shows $\tau_{\mathrm{cc}}=0.7 \tau_{\mathrm{cp}}$. Right: The cumulative distributions of the $200 \mu \mathrm{m}$ measurements and the ratio of the quantiles, $Q_{i}\left(\tau_{\mathrm{cc}}\right) / Q_{i}\left(\tau_{\mathrm{cp}}\right)$.

Another indicator of relative conditions is the ratio of the quantiles for the two locations (Fig. 10, right). For the bulk $(75 \%)$ of the measurements, this ratio is $0.7-0.75$, consistent with the regression parameters. There is no significant seasonal or diurnal variation in the ratio.

As the altitude difference between the sites $\Delta h=$ $550 \mathrm{~m}$, the $350 \mu \mathrm{m}$ optical depth ratio $\tau_{\mathrm{cc}} / \tau_{\mathrm{cp}}=$ 0.7 corresponds to an (exponential) scale height $\Delta h / \ln \left(\tau_{\mathrm{cp}} / \tau_{\mathrm{cc}}\right)=1540 \mathrm{~m}$. As discussed below ( $(6.3)$, this optical depth scale height should not be confused with the water vapor scale height.

\subsection{2. $200 \mu m$ transparency}

Under exceptionally dry conditions, observations at $200 \mu \mathrm{m}$ may be contemplated (Mankin et al. 1973; Matsushita et al.|1999; Paine et al. 2000; Blundell et al. 2002; Pardo et al. 2005; Ward-Thompson et al. 2005). One of the tippers in Chile was fitted with an additional bandpass filter so it alternates every $13 \mathrm{~min}$ between a measurement at $350 \mu \mathrm{m}$ and one at $200 \mu \mathrm{m}$. Both on the Chajnantor plateau and on Cerro Chajnantor, there is a strong correlation between successive measurements at the two wavelengths (Fig. 11). Saturation of the $200 \mu \mathrm{m}$ measurements is clearly apparent under poor conditions, when $\tau>4$. For good conditions, $\tau(350 \mu \mathrm{m}) \leq 1.55$, linear regression indicates

$$
\tau(200 \mu \mathrm{m})=(3.2 \pm 0.3) \tau(350 \mu \mathrm{m})-(0.35 \pm 0.3),
$$

with no significant difference between the two sites. This measured optical depth ratio is similar to previous determinations (Matsushita et al. 1999; 

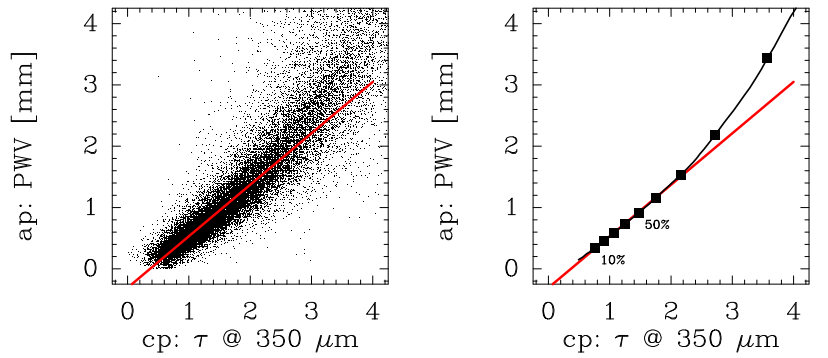

Figure 13. Left: Correlation between simultaneous measurements of the broad band $350 \mu \mathrm{m}$ zenith optical depth on the Chajnantor plateau and the precipitable water vapor (PWV) column density derived from simultaneous $183 \mathrm{GHz}$ spectroscopic measurements at APEX. Right: Quantiles of the paired measurements with the deciles marked ( $Q Q$ plot). In both panels, the guide line illustrates $\mathrm{PWV}[\mathrm{mm}]=0.84 \tau(350 \mu \mathrm{m})-0.31$.

Ward-Thompson et al. 2005).

In the absence of simultaneous $200 \mu \mathrm{m}$ measurements at the two locations, comparing the distributions of the measurements provides an indication of relative site quality despite the risk of selection bias. Under good conditions when the measurements are not badly saturated, $\tau(200 \mu \mathrm{m})<3.5$ (first quartile and better), the optical depth ratio is $70 \%$ (Fig. 12), the same as for the $350 \mu \mathrm{m}$ measurements.

Even at excellent sites, $200 \mu \mathrm{m}$ observations will be only practical under exceptional circumstances because the atmospheric transmission is otherwise so poor. On Cerro Chajnantor, for example, the first quartile zenith optical depth is 2.3 , which corresponds to $10 \%$ transmission. Exceptional observing conditions occur more often on Cerro Chajnantor than on the plateau.

\subsection{3. $183 \mathrm{GHz}$ water vapor line}

Radiometry of spectral lines, including the $183 \mathrm{GHz}$ line, is a standard technique for measuring the atmospheric water vapor content (see Askne \& Westwater 1986). Furthermore, the correspondence between water vapor and submillimeter atmospheric transparency is well established for individual sites (e.g., Matsushita \& Matsuo 2003; Pardo et al. 2004; Tamura et al. 2011). On the Chajnantor plateau, a heterodyne spectrometer was mounted on the APEX telescope in 2006 to measure the strength of the $183 \mathrm{GHz}$ line during astronomy observations. These independent measurements of the precipitable water vapor (PWV) column density are well correlated with simultaneous tipper measurements of the $350 \mu \mathrm{m}$ optical depth (Fig. 13. left). Under most (70\%) conditions, $\tau(350 \mu \mathrm{m})<2.2$, the correlation is linear but there is a significant departure from linearity under poor $(30 \%)$ conditions, indicating saturation in the $350 \mu \mathrm{m}$ measurement. The quantiles of the paired measurements provide another indication of the correlation (Fig. 13 right). Tracing the ridge along the maximum of the measurement distribution, the quantiles clearly show the linear correlation under good conditions and the curvature of the distribution under poor conditions. For $\tau(350 \mu \mathrm{m})<2.55$, linear regression indicates

$$
\mathrm{PWV}[\mathrm{mm}]=0.84 \tau(350 \mu \mathrm{m})-0.31 \text {. }
$$

Although this particular relation between PWV and $\tau(350 \mu \mathrm{m})$ is only valid in the environs of Chajnantor
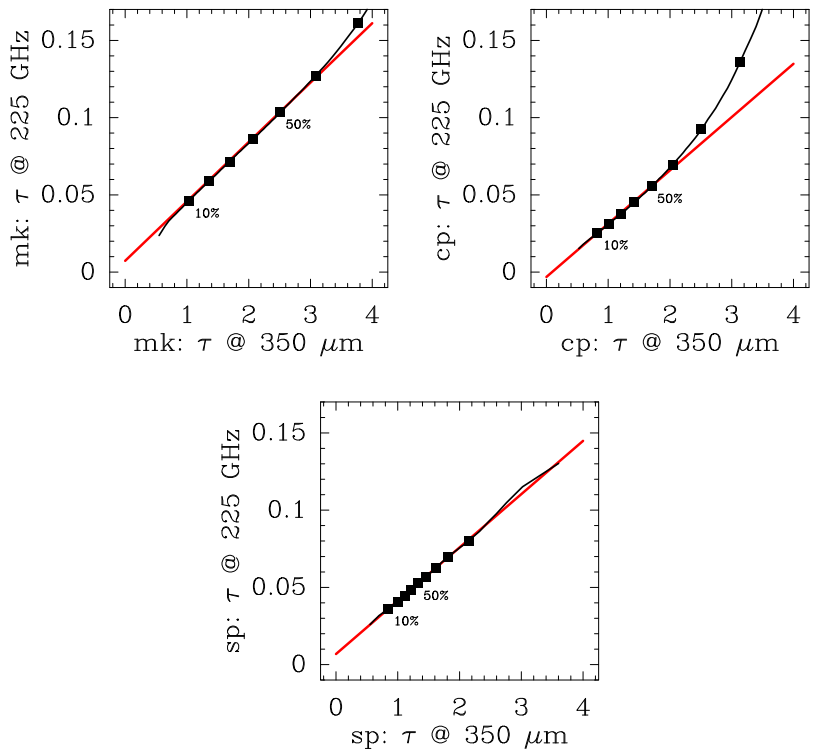

Figure 14. Quantiles of the broad band $350 \mu \mathrm{m}$ and the narrow band $225 \mathrm{GHz}$ zenith optical depths measured simultaneously on Maunakea (top left), simultaneously on the Chajnantor plateau (top right), but many years apart at the South Pole (below) ( $Q Q$ plots). Deciles are marked. The guide lines illustrate $\tau(350 \mu \mathrm{m})=$ $26 \tau(225 \mathrm{GHz})-0.2$ for Maunakea, $\tau(350 \mu \mathrm{m})=29 \tau(225 \mathrm{GHz})+0.1$ for Chajnantor, and $\tau(350 \mu \mathrm{m})=29 \tau(225 \mathrm{GHz})-0.2$ for the South Pole.

$(\S 6.2)$, the measured correlation reaffirms that the tipper data are good indicators of observing conditions at all sites.

Extrapolating the measurements to the limit of no water vapor, the optical depth would be $\tau(350 \mu \mathrm{m})=$ $0.38(=0.31 / 0.84)$. Although artifacts in the tipper measurements or in the radiometer measurements (or in both) may contribute, this zero PWV optical depth is indicative of the absorption caused by dry air (see Pardo et al. 2001a, b, 2005).

\subsubsection{5 $\mathrm{GHz}$ transparency}

Transparency measurements at or about $225 \mathrm{GHz}$ have been made to characterize observing conditions at many sites (e.g., Hogg et al. 1988; Kohno et al. 1995). On Maunakea and on the Chajnantor plateau, preexisting narrow band $225 \mathrm{GHz}$ heterodyne tippers (Radford \& Chamberlin 2000) operated simultaneously with the submillimeter tippers. At both locations, the measured broad band $350 \mu \mathrm{m}$ and narrow band $225 \mathrm{GHz}$ zenith optical depths are well correlated (Fig. 14). On Maunakea when $\tau(350 \mu \mathrm{m})<2.55$, linear regression indicates

$$
\tau(350 \mu \mathrm{m})=(26 \pm 3) \tau(225 \mathrm{GHz})-(0.2 \pm 0.2)
$$

and on the Chajnantor plateau when $\tau(350 \mu \mathrm{m})<2.05$,

$$
\tau(350 \mu \mathrm{m})=(29 \pm 3) \tau(225 \mathrm{GHz})+(0.1 \pm 0.1) .
$$

The relatively large parameter uncertainties are caused by the scatter in both sets of measurements.

At the South Pole, a $225 \mathrm{GHz}$ tipper was operated in 1992 (Chamberlin \& Bally 1994), prior to the development of the submillimeter tipper. Although the measurements were made many years apart, because the interannual consistency at the South Pole comparison of 


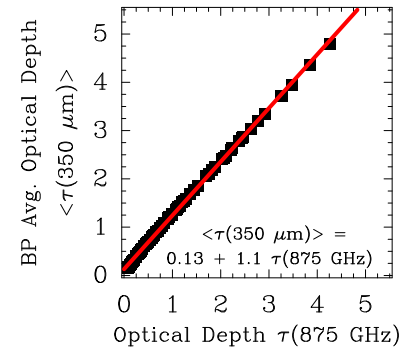

Figure 15. Predicted linear correspondence between the broad band $350 \mu \mathrm{m}$ optical depth, $\langle\tau(350 \mu \mathrm{m})\rangle$, and the narrow band $875 \mathrm{GHz}$ optical depth, $\tau(875 \mathrm{GHz})$. Markers show the predictions of ATM (Pardo et al. 2001a) for the wide range of conditions encountered at the four deployment sites, $223 \mathrm{~K}<T_{\text {base }} \leq 293 \mathrm{~K}$, $0 \mathrm{~mm} \leq \mathrm{PWV} \leq 2 \mathrm{~mm}$, and $2835 \mathrm{~m} \leq$ altitude $\leq 5000 \mathrm{~m}$. The model calculations used standard atmospheric profiles with $T_{\text {base }}$ as the ground level (base) air temperature. The solid line and equation show a linear fit to the model predictions. The particular regression coefficients depend on the reference frequency, $875 \mathrm{GHz}$ in this case.

the distribution quantiles provides an indication of the correspondence,

$$
\tau(350 \mu \mathrm{m})=29 \tau(225 \mathrm{GHz})-0.2 .
$$

At least within the parameter uncertainties, the regression slopes for the three sites are consistent and similar to previous, independent determinations near Chajnantor (Matsuo et al. 1998; Matsushita et al.|1999). The regression offsets, however, show a small but noticeable difference, corresponding to $\Delta \tau(225 \mathrm{GHz}) \approx 0.01$. This might be ascribed, at least partly, to systematic differences in the $225 \mathrm{GHz}$ tippers. Although they share a common original design (Hogg et al. 1988), they were modified and maintained independently over the years. During the period of the submillimeter measurements, the $225 \mathrm{GHz}$ tippers were never compared side by side. An incident on Maunakea suggests these instruments may be sensitive to setup conditions. In 2015 January, the alignment of the scanning mirror on the $225 \mathrm{GHz}$ tipper was damaged during an ice storm. After repairs and realignment, the correlation between subsequent $225 \mathrm{GHz}$ and $350 \mathrm{mum}$ measurements exhibited a similar slope but the offset decreased in magnitude (Radford 2016). This incident highlights the limitations of these particular $225 \mathrm{GHz}$ tippers for measurements under very dry conditions.

\section{DISCUSSION}

Recent years have seen the development of good models of radiative transfer in the atmosphere at microwave and submillimeter wavelengths, including, but not limited to, ATM (Pardo et al. 2001a) and am (Paine 2014). (For the purposes of this discussion, the predictions of ATM and $a m$ are essentially the same.) These models provide a framework for interpreting the tipper measurements but do not, of course, predict how often good conditions might occur at any site.

\subsection{Bandwidth}

Because the tippers incorporate broad band filters, the measured zenith optical depth corresponds to an average of the atmospheric transmission spectrum, $\theta(\nu)$ weighted by the filter bandpass, $F(\nu)$,

$$
\langle\tau\rangle=-\ln \left[\int \theta(\nu) F(\nu) d \nu / \int F(\nu) d \nu\right] .
$$

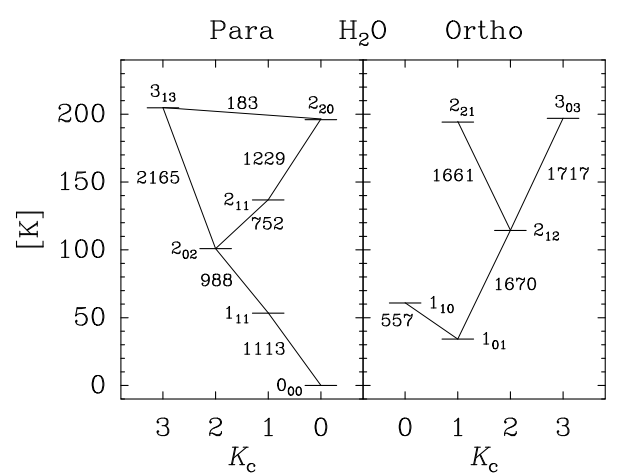

Figure 16. Energy levels of low excitation water vapor rotational states with selected dipole transition frequencies denoted in $\mathrm{GHz}$ (after Chantry 1971).

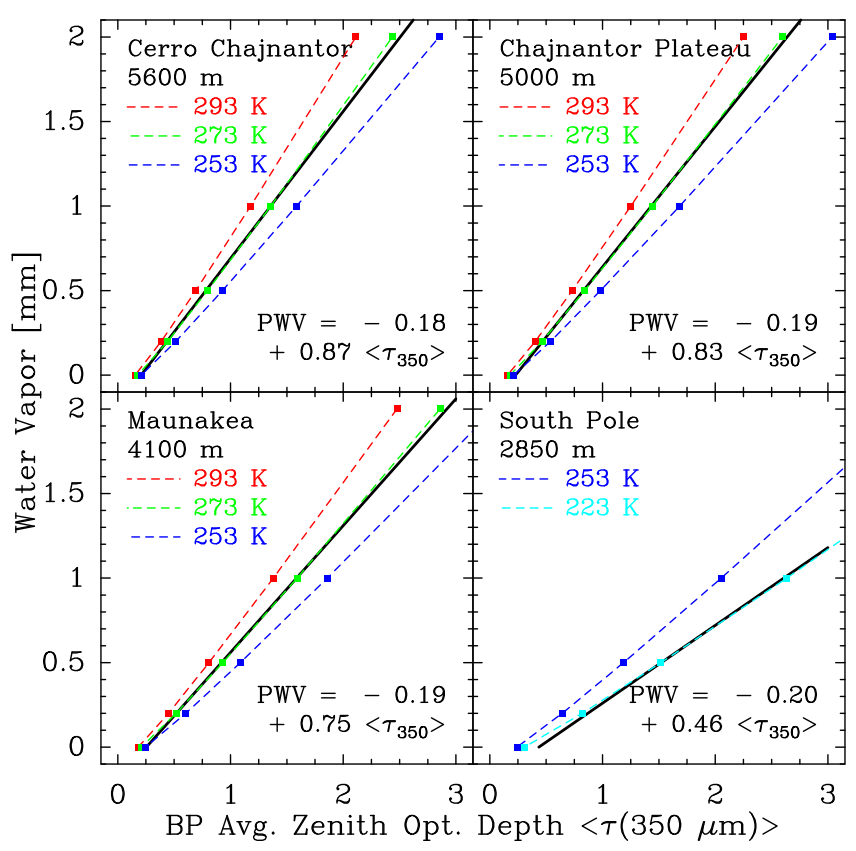

Figure 17. Predicted correspondence between broad band $350 \mu \mathrm{m}$ optical depth and precipitable water vapor (PWV) for the range of temperatures and altitudes characteristic of the four deployment sites. The markers and dashed lines show the predictions of ATM (Pardo et al. 2001a) while the solid lines and equations indicate linear fits to those model predictions for $273 \mathrm{~K}$ on Maunakea and Chajnantor and for $223 \mathrm{~K}$ at the South Pole.

The average broad band transparency is less than the peak transparency in the center of the atmospheric window, raising the question whether the broad band optical depth is a good diagnostic of conditions for narrow band observations. Model calculations with ATM (Pardo et al. 2001a) for a wide range of conditions predict, however, an almost linear relationship between the average broad band optical depth and the narrow band optical depth at a particular frequency (Fig. 15). Hence the measured broad band optical depth is a good diagnostic of conditions for both broad band and narrow band observations.

\subsection{Atmospheric Transmission and Water Vapor}

Because water vapor has such a large effect on atmospheric transmission, the precipitable water vapor 


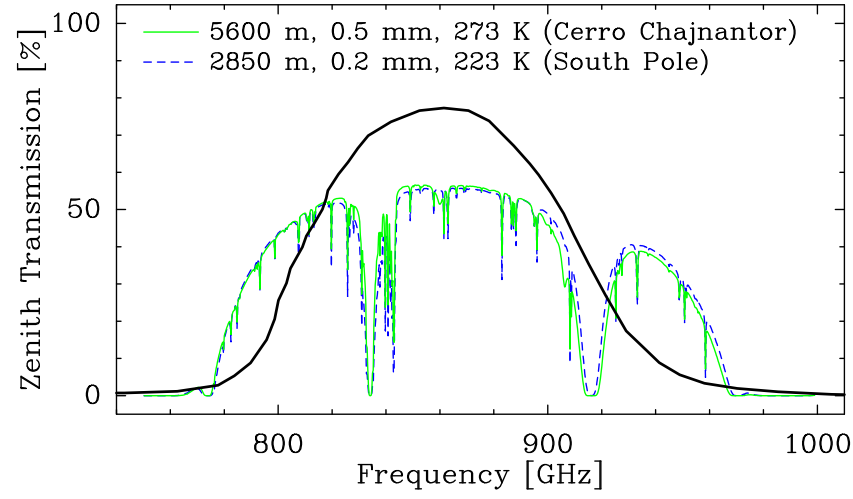

Figure 18. Atmospheric transmission across the $350 \mu \mathrm{m}$ window predicted by ATM (Pardo et al. 2001a) for good conditions at the South Pole $(0.2 \mathrm{~mm}$ PWV $)$ and on Cerro Chajnantor $(0.5 \mathrm{~mm}$ PWV). The measured transmission spectrum of the tipper's bandpass filter is overlaid (solid line).

(PWV) is often used as a metric of observing conditions, especially when considering time variations at a single site. When comparing sites with very different circumstances, however, it can be deceptive to focus solely on the PWV.

The submillimeter atmospheric windows are bracketed by strong, pressure broadened, and saturated transitions between low excitation water vapor rotational states (Fig. 16). Transmission in these windows depends primarily on three quantities: the pressure, determined by the site altitude; the air temperature; and the the water vapor column density (PWV). Models of atmospheric radiative transfer predict the windows are more transparent at higher altitude, at higher temperature, and with less water vapor (Fig. 17). The effects of water vapor and altitude (pressure) have straightforward explanations. When there is more water vapor, the lines are stronger and more saturated so the transmission is lower. At higher altitude (lower pressure), there is less pressure broadening so the line wings intrude less on the windows and the transmission is higher.

The temperature effect is perhaps less intuitive. On the one hand, saturated cold air holds less moisture than saturated warm air. In Antarctica, the absolute humidity is very low even though the very cold air in close contact with the ice sheet is almost saturated, with a typical relative humidity around $80 \%$ (Gettelman et al. 2006). At Chajnantor or Maunakea, by contrast, the best conditions occur occur when the air is unsaturated and the relative humidity is very low, around $10 \%$ or less. On the other hand, the major water vapor absorption lines link states with excitation temperatures of 50$150 \mathrm{~K}$ (Fig. 16). For the same amount of water vapor, the populations of these low excitation states will be greater at lower temperature than at higher temperature. This has the same effect as increasing the humidity: the lines are stronger and more saturated so the transmission is lower. The temperature-humidity tradeoff can be dramatic. For good winter conditions at the South Pole and on Cerro Chajnantor, a $50 \mathrm{~K}$ temperature difference is roughly equivalent to doubling the amount of water vapor (Fig. 18).

For the Chajnantor plateau, the slopes of the predicted correspondence between optical depth and PVW
Table 3

Water Vapor Scale Height

\begin{tabular}{lccccc}
\hline \hline & $\begin{array}{c}\tau(350 \mu \mathrm{m}) \\
\text { Chaj. } \\
\text { plateau }\end{array}$ & $\begin{array}{c}\text { Cerro } \\
\text { Chaj. }\end{array}$ & $\begin{array}{c}\text { PWV }[\mathrm{mm}] \\
\text { Chaj. }\end{array}$ & $\begin{array}{c}\text { Cerro } \\
\text { plateau }\end{array}$ & $\begin{array}{c}\text { Chaj. } \\
\text { scl. ht. } \\
{[\mathrm{m}]^{*}}\end{array}$ \\
\hline $75 \%$ & 2.7 & 1.9 & 2.0 & 1.3 & 1280 \\
$50 \%$ & 1.5 & 1.1 & 1.0 & 0.6 & 1080 \\
$25 \%$ & 1.0 & 0.7 & 0.53 & 0.28 & 860 \\
\hline
\end{tabular}

${ }^{*} \mathrm{WV}$ scale height $=550 \mathrm{~m} / \ln \left(\mathrm{PWV}_{\mathrm{cp}} / \mathrm{PWV}_{\mathrm{cc}}\right)$

(Fig. 17) and of the measured correlation between the tipper and $183 \mathrm{GHz}$ radiometer measurements (Fig. 13 \& Eq. 7) are very close. But the predicted offset, $-0.19 \mathrm{~mm}$, is only two thirds of the measured offset, suggesting a residual instrumental artifact in the tipper or the radiometer or both.

\subsection{Water Vapor Scale Height}

Cerro Chajnantor rises $550 \mathrm{~m}$ above the Chajnantor plateau. The measured optical depth ratio between these locations (Fig. 10) corresponds to a scale height of $1540 \mathrm{~m}$ (§5.3.1). Because of the zero water vapor offset in the optical depth measurements, however, which is at least partly due to dry air absorption, the optical depth and water vapor scale heights are not the same. Moreover, the optical depth ratio is constant, $\tau_{\mathrm{cc}} / \tau_{\mathrm{cp}}=0.7$, for the majority of the measurements. Hence the water vapor scale height is not constant, but varies with conditions: it is smaller when conditions are good.

To estimate the typical water vapor scale height, the measured optical depth ratio (Fig. 10 \& Eq. 5) may be combined with the measured correlation between the optical depth and water vapor on the Chajnantor plateau. (Fig. 13 \& Eq. 7). The resulting median ratio, $\mathrm{PVW}_{\mathrm{cc}} / \mathrm{PVW}_{\mathrm{cp}}=0.6$, corresponds to an (exponential) water vapor scale height of $1080 \mathrm{~m}$ (Table 3). The first quartile scale height is smaller, $860 \mathrm{~m}$, and the third quartile is larger, $1280 \mathrm{~m}$. This calculation ignores, however, the predicted altitude dependence of the correspondence between optical depth and water vapor (Fig. 17). If the predicted correspondence is used, rather than the measured correlation, then the estimated water vapor scale height is about $20 \%$ larger, with a median about $1300 \mathrm{~m}$.

These estimates of the water vapor scale height and PWV ratio are similar to previous results for the Chajnantor vicinity. Radiosondes launched in 1998-2000 from the Chajnantor plateau indicated a typical water vapor scale height of $1.1 \mathrm{~km}$ (Giovanelli et al. 2001). Sequential submm FTS measurements on the Chajnantor plateau in 1997-1999 and $40 \mathrm{~km}$ north on Cerro Sairecabur $(5500 \mathrm{~m})$ in 2000-2002 found a typical PWV ratio of 0.6 (Blundell et al. 2002). Simultaneous $183 \mathrm{GHz}$ water vapor line measurements on Cerro Chajnantor and at APEX in 2011 December indicated a typical PWV ratio of 0.64 (Bustos et al. 2014). Simultaneous nighttime near infrared $(1.9 \mu \mathrm{m})$ measurements on Cerro Chajnantor and $183 \mathrm{GHz}$ water vapor line measurements at APEX in 2009-2011 indicated a PWV ratio of $0.5-0.6$ (Konishi et al. 2015).

\section{SUMMARY}

Identical tipping radiometers were deployed to Maunakea, to the South Pole, and to the environs of Cerro 
Chajnantor in northern Chile to measure the broadband $350 \mu \mathrm{m}$ and $200 \mu \mathrm{m}$ atmospheric transparency. These measurements confirm all sites experience periods of excellent observing conditions.

The Chajnantor vicinity and the South Pole experience superior transparency more often than Maunakea. On the Chajnantor plateau and at the South Pole, the best conditions are similar and occur roughly the same amount of the time (25\%). At all sites, the transparency is better at night and during the winter. Seasonal and interannual variations are similar in magnitude on Maunakea whereas the seasonal variation is more pronounced at Chajnantor. The South Pole displays remarkable consistency from year-to-year.

Cerro Chajnantor enjoys significantly better transparency than the Chajnantor plateau. Most (75\%) of the time, the $350 \mu \mathrm{m}$ optical depth on Cerro Chajnantor is about $70 \%$ of the optical depth on the plateau, indicating the typical water vapor scale height is $1100-1300 \mathrm{~m}$. When conditions are good in the Chajnantor vicinity, the $200 \mu \mathrm{m}$ optical depth is 3.2 times the $350 \mu \mathrm{m}$ optical depth.

Because of seasonal and interannual variations in observing conditions and limitations on sky coverage at any one place, no location alone is adequate for all purposes. Rather it is fruitful to have telescope facilities distributed across several sites.

Many thanks to the people who contributed to this project over the years. E. Schartman constructed the instruments and initially deployed them; R. Freund designed the electronics; the South Pole winterover scientists kept the instrument running; S. Baca, E. Bufil, R. Chamberlin, A. Guyer, P. Nelson, K. Aird, E. Leitch, D. Marrone, R. Bustos, J. Cortes, C. Jara, F. Muñoz, G. Gull, C. Henderson, A. Otárola, R. Reeves, R. Rivera, and G. Valladares, provided invaluable help with deployments; S. Paine kindly measured the window transparencies; P. Ade provided some of the filters; M. Holdaway provided advice on interpretation; E. Young allowed us to use his FTS; K. Xiao did the added window experiments at the South Pole; and the referee offered several constructive comments. APEX, AST/RO, CSO, CBI, QUIET, JCMT, NRAO/ALMA, SMA, and SPT provided space, power, and network connections. APEX provides open access to their meteorological data. Access to Cerro Chajnantor was possible because the University of Tokyo constructed a road.

Development of the instruments was supported by Carnegie Mellon University and the National Radio Astronomy Observatory. Deployment to the South Pole was supported by the Center for Astrophysical Research in Antarctica, a National Science Foundation Science and Technology Center operated under cooperative agreement. The National Radio Astronomy Observatory is a facility of the National Science Foundation operated under cooperative agreement by Associated Universities, Inc. The Caltech Submillimeter Observatory (CSO) was operated by the California Institute of Technology with support from the National Science Foundation (AST0838261). CCAT site evaluation was carried out in the Parque Astronómico Atacama in northern Chile under the auspices of the Programa de Astronomía, a program of the Comisión Nacional de Investigación Científica y Tecnológica de Chile (CONICYT). CCAT site evaluation received partial support from the National Science Foundation (AST-0431503).

\section{REFERENCES}

Askne, J. I. H., \& Westwater, E. R. 1986, IEEE Trans. Geosci. \& Remote Sensing, 24, 340

Beasley, A. J. 2006, Proc. SPIE, 6267, 626702

Benford, D. J. 1998, PhD thesis, California Institute of Technology

Benford, D. J., Gaidis, \& Kooi, J. 1999, in Tenth Int. Symp. Space THz Tech., 402

Blundell, R., Barrett, J. W., Gibson, H., et al. 2002, in Thirteenth Int. Symp. Space THz Tech., 159

Burton, M. G., Cui, X., \& Tothill, N. F. H., eds. 2012, Proc. IAU, Vol. 8, Astrophysics from Antarctica, Symp. S288

Businger, S., McLaren, R., Ogasawara, R., Simons, D., \& Wainscoat, R. J. 2002, Bull. Am. Met. Soc., 83, 858

Bustos, R., Rubio, M., Otárola, A., \& Nagar, N. 2014, PASP, 126, 1126

Calisse, P. G. 2004, PASA, 21, 252

Calisse, P. G., Ashley, M. C. B., Burton, M. G., et al. 2004a, PASA, 21, 256

Calisse, P. G., Ashley, M. C. B., Burton, M. G., et al. 2004b, in The Dense Interstellar Medium in Galaxies, ed. S. Pfalzner, C. Kramer, C. Staubmeier, \& A. Heithausen, 353

Chamberlin, R., \& Bally, J. 1994, App. Opt., 33, 1095

Chantry, G. W. 1971, Submillimetre Spectroscopy (Academic Press)

Dicke, R. H., Beringer, R., Kyhl, R. L., \& Vane, A. B. 1946,

Phys. Rev., 70, 340

Dowell, C. D., Allen, C. A., Babu, R. S., et al. 2003, Proc. SPIE, 4855,73

Duncan, W. D., Robson, E. I., Ade, P. A. R., Griffin, M. J., \& Sandell, G. 1990, MNRAS, 243, 126

Erasmus, D. A. 1986, PASP, 98, 254

Gettelman, A., Walden, V. P., Miloshevich, L. M., Roth, W. L., \& B., H. 2006, J. Geophys. Res., 111, D09S13

Giovanelli, R., Darling, J., Henderson, C., et al. 2001, PASP, 113, 803

Güsten, R., Nyman, L. A., Schilke, P., et al. 2006, A\&A, 454, L13

Hemmati, H., Mather, J. C., \& Eichhorn, W. L. 1985, App. Opt., 24,4489

Hogg, D., Owen, F., \& McKinnon, M. 1988, MMA Memo 45 (NRAO)

Holdaway, M. A., Ishiguro, M., Nakai, N., \& Matsushita, S. 1996, MMA Memo 158 (NRAO)

Holland, W. S., Robson, E. I., Gear, W. K., et al. 1999, MNRAS, 303,659

Hunter, T. R. 1997, PhD thesis, California Institute of Technology

Hunter, T. R., Benford, D. J., \& Serabyn, E. 1996, PASP, 108, 1042

King, J. C., \& Turner, J. 1997, Antarctic Meteorology (Cambridge U. P.)

Kohno, K., Kawabe, R., Ishiguro, M., et al. 1995, Nobeyama Radio Obs. Tech. Report 42 (NAOJ)

Konishi, M., Motohara, K., Tateuchi, K., et al. 2015, PASJ, 67, 4

Mankin, W. G., Eddy, J. A., MacQueen, R. M., Lee, R. H., \& Querfeld, C. W. 1973, Nature Physical Science, 245, 8

Marrone, D. P., Blundell, R., Tong, E., et al. 2005, in Sixteenth Int. Symp. Space THz Tech., 64

Marrone, D. P., Battat, J., Bensch, F., et al. 2004, ApJ, 612, 940

Matsuo, H., Sakamoto, A., \& Matsushita, S. 1998, PASJ, 50, 359

Matsushita, S., \& Matsuo, H. 2003, PASJ, 55, 325

Matsushita, S., Matsuo, H., Pardo, J. R., \& Radford, S. J. E. 1999, PASJ, 51, 603

Messerli, B., Grosjean, M., Bonani, G., et al. 1993, Mountain Research \& Development, 13, 117

Morrison, D., Murphy, R. E., Cruikshank, D. P., Sinton, W. M., \& Martin, T. Z. 1973, PASP, 85, 255

Padin, S., Cartwright, J. K., Mason, B. S., et al. 2001, ApJ, 549, L1

Paine, S. 2014, SMA Tech. Memo 152 vers. 8.0 (SAO) 
Paine, S., Blundell, R., Papa, D. C., Barrett, J. W., \& Radford, S. J. E. 2000, PASP, 112, 108

Pardo, J. R., Cernicharo, J., \& Serabyn, E. 2001a, IEEE Trans. Ant. Prop., 49, 1683

Pardo, J. R., Serabyn, E., \& Cernicharo, J. 2001b, J. Quant. Spectroscopy \& Radiative Transfer, 68, 419

Pardo, J. R., Serabyn, E., Wiedner, M. C., \& Cernicharo, J. 2005, J. Quant. Spectroscopy \& Radiative Transfer, 96, 537

Pardo, J. R., Wiedner, M. C., Serabyn, E., et al. 2004, ApJS, 153, 363

Peterson, J. B., Radford, S. J. E., Ade, P. A. R., et al. 2003, PASP, 115, 383

Peterson, J. B., \& Richards, P. L. 1984, Int. J. Infrared Millimeter Waves, 5, 1507

Putley, E. H. 1980, in Optical and Infrared Detectors, 2nd edn., ed. R. J. Keyes (Springer-Verlag), 71

Radford, S. 2002, ASPCS, 266, 148
Radford, S. J. E. 2011, RevMexAA (SC), 41, 87

Radford, S. J. E. 2016, SMA Tech. Memo 164 (SAO)

Radford, S. J. E., \& Chamberlin, R. A. 2000, ALMA Memo 334.1 (NRAO)

Radford, S. J. E., Giovanelli, R., Gull, G. E., \& Henderson, C. P. 2008, Proc. SPIE, 7012, 70121Z

Radford, S. J. E., \& Holdaway, M. A. 1998, Proc. SPIE, 3357, 486

Tamura, Y., Kawabe, R., Kohno, K., et al. 2011, PASJ, 63, 347

Turner, D. D., Ferrare, R. A., \& Brasseur, L. A. 2001, Geophys. Res. Lett., 28, 4441

Ward-Thompson, D., Ade, P. A. R., Araujo, H., et al. 2005, MNRAS, 364, 843

Welford, W. T., \& Winston, R. 1989, High Collection Nonimaging Optics (Academic Press)

Wolter, K., \& Timlin, M. S. 2011, International Journal of Climatology, 31, 1074 